\title{
The complex Chiari malformation: an evolution of understanding
}

\author{
Douglas Brockmeyer
}

Received: 11 February 2014 / Accepted: 28 April 2014 / Published online: 5 June 2014

(C) Springer-Verlag Berlin Heidelberg 2014

In the article, "Magnetic Resonance Imaging Features of Complex Chiari Malformation Variant of Chiari 1 Malformation," Moore and Moore [1] describe the radiologic findings associated with a variation of the typical Chiari 1 disorder. They examined 151 patients identified with Chiari 1 malformation from a large imaging database and subclassified them as either "standard Chiari 1 malformation" or "complex Chiari malformation." Various radiologic parameters were then analyzed in a standardized fashion in each subcategory. The authors found that obex level was significantly associated with the complex Chiari malformation type and that complex Chiari malformation was strongly associated with more severe craniocervical pathology in general, such as a kyphotic craniocervical angle. Further analysis revealed that patients in the complex Chiari malformation group had a significant chance of requiring more frequent and complex surgical interventions than their Chiari 1 malformation counterparts.

Although the sine qua non of Chiari 1 malformation is cerebellar tonsillar ectopia greater than $5 \mathrm{~mm}$ below the foramen magnum, it is well known that clinical and radiologic variants of the malformation exist. For example, previous authors have described the presence of Chiari 0 and Chiari 1.5 malformations, both thought to be variations on the Chiari hindbrain abnormality $[2,3]$. Therefore, continuing to describe a highly variable and complex radiologic skull base/hind brain abnormality with the simplistic moniker of "Chiari 1 malformation" intuitively makes no sense. Our previous work [4] initially describing the "complex Chiari malformation," was born out of the clinical observation that more extensive and complex surgical procedures are required

D. Brockmeyer $(\bowtie)$

Pediatric Neurosurgery, University of Utah,

Primary Children's Medical Center, 100 N. Mario Capechhi Drive,

Salt Lake City, UT 84113, USA

e-mail: Douglas.brockmeyer@hsc.utah.edu to alleviate symptomatology in patients with complex radiologic findings, such as odontoid retroflexion, basilar invagination, and Chiari 1.5 brainstem displacement. These procedures include occipitocervical fusion, odontoid reduction and sometimes anterior transoral or transnasal odontoid resection. In contrast, patients with traditional Chiari 1 pathology very rarely, if ever, require more than one decompressive surgical procedure.

In commenting on the paper by Moore and Moore [1], I feel I must clarify that what the authors describe as a "complex Chiari malformation" is really a Chiari 1.5 malformation. Therefore, what they show is that patients with a Chiari 1.5 malformation are significantly more likely to have a lower obex, basilar invagination and a more kyphotic craniocervical angle. Again, this knowledge has been previously published, although not in the neuroradiology literature.

Unfortunately, I must point out a few shortcomings of this paper. The first is that the authors do not explicitly describe the methods by which they measured the clivoaxial angle. They only state that it is "measured as the angle between lines drawn along the clivus and axis of $\mathrm{C} 2$ body/odontoid." This is critical because there is disagreement even among neurosurgeons as to how to reliably measure the clivoaxial angle. This disagreement stems from the considerable variation in the anatomical appearance of the clivus from patient to patient. Presently, our neurosurgical group uses the method described in our paper [4], in which the clivoaxial angle is defined by the angle created by a line drawn along the inferior half of the clivus and a second line intersecting the tip of the odontoid and the midportion of the inferior border of the $\mathrm{C} 2$ body. This line can be reliably reproduced between trained observers, with interclass correlation coefficients in our paper of 0.63 [4]. The other shortcoming is the lack of a standardized definition of "odontoid retroflexion" in the paper. Which angles or measurements were used in making that determination? Or is it an overall gestalt appearance of odontoid 
orientation? A recent paper in the adult neurosurgical literature addressed this issue in a comprehensive way [5]. Further explanation of these points would strengthen the paper as a whole.

Those issues aside, it is clear from this discussion that one of the most pressing problems ahead of us is to produce a standardized set of measurements related to skull base and Chiari 1 pathology. Creating precise definitions of the radiologic parameters described in this paper, including the clivoaxial angle, would go a long way in heightening awareness and assisting health care providers caring for patients with Chiari 1 malformation. Such definitions would give us insight into the pathophysiology of the Chiari disorder and might ultimately help us determine which patients would benefit most from surgical intervention. Neurosurgeons and neuroradiologists need to speak the same language when describing radiologic Chiari-related pathology, and that language needs to be firmly based in precise radiologic and clinical awareness. Ultimately, I believe that we are just beginning to understand the complex craniocervical interrelationships that exist in patients with Chiari 1 malformation and complex Chiari malformation. Skull base morphology, hydrodynamics and biomechanics all play a part in Chiari pathophysiology — we just need to determine which factors play which roles in which patients. Only further work will clarify these issues, and together with other physicians, I look forward to using that knowledge to benefit the patients we are entrusted to care for.

Conflicts of interest None

\section{References}

1. Moore HE, Moore KR (2014) Magnetic resonance imaging features of complex Chiari malformation variant of Chiari 1 malformation. Pediatr Radiol. doi:10.1007/s00247-014-3021-1

2. Tubbs RS, Iskandar BJ, Bartolucci AA et al (2004) A critical analysis of the Chiari 1.5 malformation. J Neurosurg 101:179-183

3. Chern JJ, Gordon AJ, Mortazavi MM et al (2011) Pediatric Chiari malformation type 0: a 12-year institutional experience. J Neurosurg Pediatr 8:1-5

4. Bollo RJ, Riva-Cambrin J, Brockmeyer MM et al (2012) Complex Chiari malformations in children: an analysis of preoperative risk factors for occipitocervical fusion. J Neurosurg Pediatr 10:134-141

5. Khaleel ZL, Besachio DA, Bisson EF et al (2014) Estimation of odontoid process posterior inclination, odontoid height, and $\mathrm{pB} \mathrm{C2}$ line in the adult population. J Neurosurg Spine 20:172-177 